\title{
LOW-MASS VERSUS HIGH-MASS STAR FORMATION
}

\author{
T. W. HARTQUIST
}

Max-Planck-Institute für extraterrestrische Physik, D-85740 Garching, Germany

\author{
AND \\ J. E. DYSON \\ Department of Physics and Astronomy, \\ The University of Leeds, \\ Leeds. LS2 9JT, UK
}

\begin{abstract}
Structures like the clumps identified in the CO maps of the Rosette Molecular Cloud and the dense cores such as those in B5, a cluster of cores and young low-mass stars, are key to considerations of star formation. Whether star formation is a self-inducing process or one that causes itself to turn off depends greatly on whether the responses of the interclump and intercore media to young stars cause the collapse of clumps or cores to be faster than their ablation. We present a naive introduction to the lengthscales over which such responses are significant, mention ways in which the responses might induce collapse, review some of the little that is known of how flows of media around clumps and cores ablate them, and then return to the issue of the lengthscales over which such responses are significant by considering the global properties of mass-loaded flows in clumpy star forming regions.
\end{abstract}

\section{Introduction}

Regions of recent and/or ongoing high-mass star formation are amongst the most brilliant diffuse optical sources in the sky, in contrast to regions in which only low-mass stars are born. The dramatic nature of the response of the interstellar medium around high-mass stars and work on the ages of massive stars in Orion led eventually to the realisation that that type of 
response induces further massive stars to form and that such stars can be produced sequentially (Elmegreen \& Lada 1977).

In contrast, the formation of a low-mass star is generally treated as a process occurring in isolation (e.g. Mouschovias 1987; Shu, Adams, \& Lizano 1987), an approach which may be valid in many cases. However, young low-mass stars are sources of radiation and winds sufficiently powerful to induce significant responses in the interstellar matter near them, and we must address the possibility that low-mass star formation is often self-regulating.

Much of the issue of differences between and similarities of high-mass and low-mass star formation is, therefore, concerned with the ways in which gas in an interstellar cloud responds to the formation of a high-mass star or the birth of a low-mass star. In section 2 we consider briefly the lengthscales on which the responses to low-mass and high-mass stars are significant. The ways in which clumps react to a newly formed nearby star are important in determining whether star formation induces further stellar birth; in sections 3 and 4 respectively, we mention mechanisms by which clump collapse might be triggered or retarded by a star. Section 5 returns to the topic of section 2 and contains comments about what is known of how the clumpiness of a medium might alter its global response to stellar sources. Section 6 concludes the review.

\section{The Scale of Response}

Cioff, McKee, \& Bertschinger (1988) have shown that a supernova occurring in a uniform non-magnetic medium will increase the pressure of the surrounding medium within a radius $R_{\mathrm{SN}}$, given by

$$
R_{\mathrm{SN}} \simeq 70 E_{51}^{31 / 98} n_{\mathrm{H}}{ }^{-18 / 49}\left\{\frac{\beta C_{1}}{10 \mathrm{~km} \mathrm{~s}^{-1}}\right\}^{-3 / 7} \mathrm{pc} .
$$

$E_{51}, n_{\mathrm{H}}, C_{1}$, and $\beta$ respectively are the supernova energy in units of $10^{51}$ $\mathrm{erg}$, the hydrogen nuclei number density in $\mathrm{cm}^{-3}$, the sound speed of the ambient medium, and a constant near unity. The use of equation (1) to estimate the lengthscale $R_{\text {SNGMC }}$ over which a supernova will have a significant effect in a giant molecular cloud (GMC) is naive because a cloud is magnetized and clumpy. Even so, to make a rough estimate of $R_{\mathrm{SNGMC}}$, we will use $R_{\mathrm{SN}}$, but to do so we must first specify $n_{\mathrm{H}}$ and $C_{1}$. We assume that the supernova occurs in the interclump medium of a GMC of which the Rosette Molecular Cloud (RMC) is a particularly well-studied example. Williams, Blitz, \& Stark (1995) argued that the mean interclump neutral atomic number density in the RMC is $4 \mathrm{~cm}^{-3}$. Williams et al. gave the mean $\mathrm{H}_{2}$ number density in clumps as about $220 \mathrm{~cm}^{-3}$ and found the 
velocity dispersions in the clumps to be $2-3 \mathrm{~km} \mathrm{~s}^{-1}$ typically. For an interclump medium of atomic hydrogen with a number density of about $4 \mathrm{~cm}^{-3}$ to confine such a clump (which seems required because according to Williams et al., a good fraction of the clumps in the RMC are not massive enough to be bound by the components of the gravitation field that they generate) the effective interclump sound speed must be roughly $30 \mathrm{~km} \mathrm{~s}^{-1}$ (which may be the case if the interclump magnetic field is of the corresponding strength). For $n_{\mathrm{H}} \simeq 4 \mathrm{~cm}^{-3}$ and $C_{1} \simeq 30 \mathrm{~km} \mathrm{~s}^{-1}$, equation (1) gives $R_{\mathrm{SN}} \simeq 26 E_{51}^{31 / 98} \beta^{-3 / 7} \mathrm{pc}$, a particularly interesting result because it suggests that a single supernova can affect the pressure over a reasonable fraction, but not all, of a GMC. If it were to cause a large increase in the pressure over a distance small compared to the typical distance between clumps in which high-mass stars form one might expect high-mass star formation not to be self-triggered at all. If a single supernova could cause a large increase in the pressure through the interclump medium of an entire GMC, one might expect a burst of high-mass star formation over the entire GMC rather than high-mass star formation to be sequential.

The ${ }^{13} \mathrm{CO}$ and ${ }^{12} \mathrm{CO}$ observations of the RMC that Williams et al. analysed do not readily lead to the discovery of dense cores, which are more easily identified from $\mathrm{NH}_{3}$ data but can be mapped in $\mathrm{CO}$ emissions (e.g. Myers 1990). Young low-mass stars are associated with about half of the dense cores indicating that they are often progenitors of stars. Those in which low-mass stars form usually have molecular hydrogen number densities of about $10^{4}-10^{5} \mathrm{~cm}^{-3}$ and masses within a factor of a few of $10 \mathrm{M}_{\odot}$. While some cores are more isolated, a sizeable fraction are found to exist in clusters somewhat like Barnard 5 (B5). The CO map of B5 of Goldsmith, Langer, \& Wilson (1986) shows the presence of 5 dark cores as well as the locations of young stellar objects which are IRAS sources. The CO data provide clear evidence that the winds of the young stars affect the dynamics of the intercore medium of the B5 core cluster on scales comparable to the intercore separations. Obviously, at least in some cases, the formation of a low-mass star cannot be considered to be occurring in isolation. Rather, sometimes on smaller scales and during later stages of collapse the births of low-mass stars affect subsequent low-mass star formation in manners that may be similar to or may contrast interestingly with the ways in which the births of high-mass stars affect subsequent high-mass star formation. The degree of similarity or contrast most likely has very much to do with the nature of the responses of clumps and of dense cores, to flows around them driven by the stars. 


\section{Triggering Clump or Core Collapse}

Mouschovias \& Spitzer (1976) showed that gravitational collapse of a magnetized clump will occur only if its mass exceeds a critical mass $M_{\mathrm{c}}$ and the external pressure is greater than a minimum value $P_{\mathrm{m}}$. For a clump with the same distribution of mass on magnetic field lines as a spherical clump of uniform density and with a constant magnetic field

$$
M_{\mathrm{c}}=\frac{0.53}{\pi}\left\{\frac{5}{9 G}\right\}^{1 / 2} \Phi
$$

and

$$
P_{\mathrm{m}}=\frac{1.89 C_{\mathrm{cl}}^{8}}{G^{3} M_{\mathrm{cl}}^{2}\left(1-\left(M_{\mathrm{c}} / M_{\mathrm{cl}}\right)^{2 / 3}\right)^{3}}
$$

where $M_{\mathrm{cl}}$ is the clump mass, $C_{\mathrm{cl}}$ is the isothermal sound speed in the clump, $\Phi$ is the magnetic flux through the clump, and $G$ is the gravitational constant. Low-mass star formation is generally supposed to occur in clumps in which initially $M_{\mathrm{cl}}<M_{\mathrm{c}}$ but ambipolar diffusion gradually reduces $\Phi$ until, eventually, $M_{\mathrm{cl}} \geq M_{\mathrm{c}}$ (e.g. Mouschovias 1987; Shu et al. 1987). Highmass star formation is more likely triggered in clumps in which $M_{\mathrm{cl}}>M_{\mathrm{c}}$ by an increase in external pressure (e.g. Elmegreen \& Lada 1977; Mouschovias 1987; Shu et al. 1987) to a value above $P_{\mathrm{m}}$ from one that is initially below $P_{\mathrm{m}}$.

Since the RMC clumps identified by Williams et al. (1995) have masses varying by over two orders of magnitude and average number densities that are restricted (perhaps, due to the sensitivity of $\mathrm{CO}$ observations to the range of measured densities) to within about a factor of four of the mean, some RMC clumps probably are magnetically supercritical (i.e. have $M_{\mathrm{cl}}>M_{\mathrm{c}}$ ) while others are magnetically subcritical (i.e. have $M_{\mathrm{cl}}<M_{\mathrm{c}}$ ). In the standard view of low-mass star formation, a core in B5 is either magnetically subcritical but becoming marginally supercritical due to ambipolar diffusion or is marginally supercritical. An increase of the pressure in the RMC interclump medium or the B5 intercore medium caused by a star compresses the clumps or the cores but only in the RMC could it possibly directly induce the gravitational instability of some (but certainly not all) objects.

In the last sentence we used the phrase 'possibly . . . induce' rather than simply 'induce' because even a huge increase of the external pressure of a magnetically supercritical cloud will not induce it to collapse if $C_{\mathrm{cl}}$ is a too sensitively increasing function of the external pressure (c.f. equation (3)). The turbulent pressure within a typical RMC clump exceeds the thermal pressure by more than an order of magnitude, and a characteristic speed associated with the turbulence should replace the isothermal sound speed in 
equation (3) when it is employed in considerations of RMC clump stability. The turbulence within an RMC clump probably consists of a superposition of Alfvén waves (e.g. Arons \& Max 1975; Mouschovias \& Psaltis 1995), but the excitation mechanism remains unknown implying that the dependence upon the external pressure of the characteristic speed associated with the turbulence to be used in equation (3) is also unknown. In our view, until this excitation mechanism is identified, the theory of sequential high-mass star formation and the theory of starbursts in external galaxies remain distant objectives (Hartquist, Dyson, \& Williams 1997).

However, knowledge of some of the mechanisms damping the waves comprising the turbulence does exist. One of importance is the friction between ions and neutrals which is easily analysed for small amplitude waves superposed on a uniform medium (Kulsrud \& Pearce 1969). The damping rate due to ion-neutral friction is inversely proportional to the fractional ionisation for waves of fixed angular frequency as long as that angular frequency is much less than the inverse of the time required for collisions with ions to transfer to a neutral particle a momentum of magnitude comparable to the neutral particle's initial momentum. Hence, the ionization structure of an RMC clump is likely of importance in determining the properties of the turbulence in it.

Hartquist et al. (1993) have suggested that $\mathrm{S}^{+}$is the most abundant ion throughout RMC clumps that do not contain infrared sources (the presence of which indicates that the clumps have collapsed). Because the simple hydrogen abstraction reactions $\mathrm{SH}^{+}+\mathrm{H}_{2} \rightarrow \mathrm{SH}_{2}^{+}+\mathrm{H}$ and $\mathrm{SH}_{2}^{+}+\mathrm{H}_{2} \rightarrow \mathrm{SH}_{3}^{+}+\mathrm{H}$ are slow at low temperatures and the ionization potential of $\mathrm{S}$ is lower than that of $\mathrm{C}$, gas phase sulphur remains primarily in $\mathrm{S}^{+}$at several times greater visual extinction than that to which carbon remains in $\mathrm{C}^{+}$. Hartquist et al. proposed that the RMC clumps in which stars have formed have at one time or another been compressed sufficiently for their visual extinctions to exceed a critical visual extinction above which most of the sulphur in them has become neutral, whereas the RMC clumps have not been compressed sufficiently exceed this critical value. In clumps in which the sulphur is primarily neutral, the fractional ionization is probably one to two orders of magnitude lower than that in clumps in which most gas phase sulphur is in $\mathrm{S}^{+}$. Thus, in the former class of clumps, the ion-neutral frictional damping of turbulence is much more rapid than in the latter class of clumps, probably resulting in the reduction of the turbulent pressure in a clump belonging to the former class and its collapse primarily along the magnetic field lines in it; of course, some collapse perpendicular to the field lines also occurs. Thus, high-mass star formation may (in a case in which the clump is initially supported in part by turbulence and is magnetically supercritical) be induced directly by an increase in the interclump pressure (in response 
to a recently formed star) if the pressure increase gives rise to a sufficient rise in visual extinction that the fractional ionization at the clump centre drops significantly. Of course, partial collapse of a magnetically subcritical and initially turbulent clump may be induced in the same way, but ambipolar diffusion must then act for star formation to occur leading probably to low-mass star formation.

The principal ions at the centres of the cores in an object like B5 are most likely $\mathrm{Mg}^{+}, \mathrm{Na}^{+}$, and molecular ions, and compression by the increase of the pressure in the intercore medium probably does not alter the fractional ionization radically (i.e. by more than a factor of a couple or a few). In addition, in each of many dense cores, the line widths of emissions indicate that the turbulent pressure is negligible compared to the thermal pressure (Myers \& Benson 1983). Therefore, the specific sort of induced collapse picture presented above for RMC clumps does not apply to dense cores.

Though we have expressed reservations above about the idea that the compression of a dense core causes major changes in the fractional ionization, the possibility that desorption of elements heavier than helium from grains ceases abruptly once a critical visual extinction (differing from the critical visual extinction mentioned above in the consideration of sulphur chemistry and varying from one star forming region to another) has been exceeded, has been advanced (Williams, Hartquist, \& Whittet 1992). Freezeout of heavy elements (and in particular, sodium, magnesium and other heavy metals) may have a major effect on the fractional ionization. When the fractional ionization is much above about $10^{-8}$, grain-neutral drag is negligible and the ambipolar diffusion time scale of a magnetically subcritical core is proportional to the fractional ionization (e.g. Mouschovias 1987). Unfortunately, the desorption and freeze-out of elements in cores is not understood, and until it is, a number of important issues concerning ionization structure and ambipolar diffusion in dense cores and the possibility that low-mass star formation may be sped up significantly by an increase in the pressure of the intercore medium, will also remain unclear.

\section{Ways in Which Star Formation May Hinder Further Star For- mation}

As mentioned at the end of the previous section, the time scale for ambipolar diffusion, important for the formation of stars in clumps and cores that are initially magnetically subcritical, is proportional to the fractional ionization. Oppenheimer \& Dalgarno (1974) and subsequently Elmegreen (1979) and Umebayashi \& Nakano (1980) have shown that at high visual extinctions where ionization is induced primarily by cosmic rays, the most 
abundant ions are those of heavy metallic atoms (e.g. $\mathrm{Na}^{+}$and $\mathrm{Mg}^{+}$) but that most of the heavy metallic atoms may be neutral. McKee (1989) has argued that the radiation of a young low-mass star in a core cluster like B5 can penetrate a dense core sufficiently to maintain a sizeable fraction of the gas phase metallic atoms in ionized states. Therefore, the birth of a low-mass star in a core cluster may retard the formation of other low-mass stars by causing an increase in the ambipolar diffusion time scale.

As seen in Goldsmith et al. (1986), the winds of the young stars in B5 move material around on lengthscales comparable to the intercore separations, and as the cores undergo collapse they are also ablated by the stellar winds. Further stellar birth would not occur if the collapse time scale were long compared to the ablation time scale.

Nittmann, Falle, \& Gaskell (1982) argued that the evaporation of a non-magnetic clump initially bound by the pressure of a more diffuse static non-magnetized medium through which a hypersonic shock passes, takes place within several clump sound crossing times. The results of the numerical simulations of Klein, McKee, \& Colella (1994) are consistent with those arguments and the earlier simulations. Evaporation is reduced if the compression causes the clump to become Jeans unstable (c.f. Woodward 1976). Evaporation also occurs but at a lowered rate if the flow in the medium surrounding the clump is subsonic in the frame of the clump (in which the Mach number of the tenuous medium's flow is measured to be $M_{\mathrm{T}}$ ). Hartquist et al. (1986) have estimated the timescale for the mass of the clump to be incorporated into the wind to be of the order of the sound crossing timescale multiplied by the cube root of the ratio of the sound speeds in the surrounding medium and the clump and divided by $M_{\mathrm{T}}^{5 / 3}$; the timescale on which the coherence of the clump is destroyed may be smaller by a factor of the ratio of the sound speeds in the clump and surrounding medium divided by $M_{\mathrm{T}}$. Clearly, if $M_{\mathrm{T}}$ is not considerably less than unity, the ambipolar diffusion timescale is much longer than the sound crossing timescale, and these estimates of evaporation timescales are relevant, then initially magnetically subcritical dense cores will be ablated before ambipolar diffusion allows them to collapse due to gravity. (The numerical simulations of MacLow et al. (1994) of shocks in magnetized media overrunning magnetized clumps suggest that the evaporation timescales appropriate for non-magnetized media and clumps may underestimate those relevant when magnetic fields are important.)

The timescale for ionization equilibrium to be reached in clumps is short compared to any dynamical timescale, and the damping timescale is likely to be less than the sound crossing timescale if $\mathrm{S}^{+}$is not a significantly abundant ion. Thus, ablation is probably less important in the collapse of magnetically supercritical RMC clumps induced by an increase in external 
pressure and in high-mass star formation than it is in low-mass star formation from magnetically subcritical dense cores. If this occurs throughout a region of star formation, an IMF biased towards more massive stars may obtain.

We would like to study the ablation of clumps and cores observationally. TMC-1 is a ridge of dense cores with a young low-mass star near the core thought to be the densest. That core has a measured $\mathrm{H}_{2}$ number density of about $5 \times 10^{5} \mathrm{~cm}^{-3}$ (Hirahara et al. 1992). The interaction of the stellar wind with it creates a turbulent boundary layer with unknown properties. Charnley et al. (1990), Nejad \& Hartquist (1994), and Rawlings \& Hartquist (1997) attempted to identify atomic and molecular diagnostics at such a boundary layer. The models of Charnley et al. (1990) and of Nejad \& Hartquist (1994) are based on the assumptions that a boundary layer is isobaric and that $\mathrm{H}^{+}$and $\mathrm{He}^{+}$from shocked stellar wind and heat are injected into core gas at specified rates. The models of Rawlings \& Hartquist (1997) are based on the assumptions that the boundary layer is isobaric and that diffusive transport between the core and the wind takes place in the boundary layer; for an optically thin boundary layer the free parameter is the diffusion timescale and the molecular, atomic, and ionic densities are specified to be appropriate for a shocked stellar wind and a dense core on either side of the boundary layer. The chemistry in all their models was restricted to simple species. An observation of $\mathrm{CO}(\mathrm{J}=6 \rightarrow 5)$ emission towards core B (in the Hirahara et al. (1992) nomenclature) in TMC-1 would serve as a useful diagnostic of the thermal structure of the boundary layer. Observations of $\mathrm{CH}$ and $\mathrm{OH}$ emissions with Arecibo might yield useful data on whether diffusive mixing in the boundary layers occurs. (Because the boundary layers are magnetized, mixing of stellar wind ions with dense core neutrals to the extent that they react together chemically might be negligible, but the issue is difficult to address theoretically.) A major problem is that a boundary layer may contain only a few percent of the total material of a core so that emissions arising in it might be hard to detect against the core emissions; this difficulty makes the existing data for CI emission from TMC-1 (Schilke et al. 1995) irrelevant for boundary layer diagnosis even though $\mathrm{C}^{0}$ and $\mathrm{C}^{+}$could acquire high fractional abundances in a boundary layer if mixing is thorough.

However, the fact that CI emission is detectable from a dense core may imply that mixing does take place in boundary layers in low-mass star forming regions (Williams, Hartquist, \& Caselli 1996; Rawlings \& Hartquist 1997; references therein). For the moment assume that dense cores form when magnetically subcritical RMC clumps dissipate their turbulence and undergo partial collapse. Williams et al. (1995) have concluded that only a few percent of the hydrogen in a clump in the RMC is not in molecular 
form; at RMC clump densities, the $\mathrm{H}_{2}$ formation time exceeds the chemical timescale on which most carbon is converted to $\mathrm{CO}$ and in addition $\mathrm{H}_{2}$ shields $\mathrm{CO}$ from photodissociation (e.g. van Dishoeck 1987) so that nearly all the carbon in an RMC clump may be contained in CO. Rawlings \& Hartquist (1997) argued against clump-scale diffusive mixing being a means of maintaining high $\mathrm{C}$ abundances in clumps or cores, and the low atomic hydrogen to $\mathrm{H}_{2}$ abundances in the RMC clump provides some evidence that clump-scale diffusion mixing is chemically unimportant.) Thus, collapse to form a dense core from a clump rich in atomic carbon on a timescale short compared to the chemical timescale can be ruled out as the source of atomic carbon in dense cores. However, CI emission can arise from a dense core in a core cluster if mixing of ions and neutrals occurs in the boundary layer between shocked stellar wind and a dark core of a previous generation and the ablated material - stellar wind mixture passes through a low enough speed termination shock and then undergoes collapse to form a new generation of cores on a timescale short compared to the chemical timescale $\left(\simeq 10^{6}\right.$ years) required to convert almost all carbon to $\mathrm{CO}$. We suggest that $\mathrm{Cr}$ emission and cyanopolyyne emission (cyanopolyyne formation is rapid when atomic carbon and simple carbon bearing molecules are simultaneously abundant) from dense cores implies that ablation of dense cores is significant in some regions and may prevent some cores from forming stars and that multiple generations of core formation occur in some regions.

The issue of the relative importance of photoabsorption versus purely mechanical effects makes the interpretation of some observed stellar windclump interactions in regions of high-mass star formation somewhat uncertain. Observations of cometary globules containing young low-mass stars in Orion show many of them to have long thin pointy tails (Sutherland et al. 1997). One possibility is that the tails form as the shocked wind of a nearby high-mass star flows subsonically past the clumps (Dyson, Hartquist \& Biro 1993); if this mechanism is responsible for their formation, spectroscopic observations made with HST or the best ground-based adaptive optics systems should lead eventually to a great deal of insight into windclump interactions in star forming regions. Alternatively, a tail may form if a D-type ionization front drives a small amount of material from the parts of the clump initially most distant from the line connecting the clump's centre of mass and the star towards the back of the clump (that side away from the star) and towards that line. This material is then shadowed by the main part of the clump as it flows away from the star to make a tail. The numerical simulations performed to demonstrate tail formation as a consequence of photoionization (Lefloch \& Lazareff 1994; 1995) include no diffuse component of the radiation field, and until simulations including 
a reasonable diffuse component are performed, we will remain somewhat sceptical about whether this mechanism actually forms tails.

The subject of the photoevaporation of clouds into a surrounding medium has a long history. However, so far, the photoevaporation of magnetized clumps has received little attention. Redman et al. (1997) have shown that the density jump across a D-critical ionization front is significantly reduced compared to the non-magnetic case when the ratio of the magnetic pressure to the gas pressure in the gas ahead of the IF exceeds a factor of about unity. The consequences for the evaporation rates of clumps requires a selfconsistent treatment analogous to those carried out for non-magnetized clumps (Dyson 1968; Kahn 1969; Bertoldi \& McKee 1990).

Other examples of clumps being affected by the winds of high-mass stars are the clumps in the Orion Kleinmann - Low region producing much of the $\mathrm{H}_{2}$ emission there (Tedds et al. 1996). The absence of double peaks in the line profiles implies that the $\mathrm{H}_{2}$ emission is probably not arising in the interclump material as it passes through bow shocks around the clumps unless the clumps are themselves highly fragmented (which appears unlikely from the observational data). However, the great symmetry of the line profiles about a central velocity seems inconsistent with the emission arising in gas being ablated from the clumps. The $\mathrm{NH}_{3}$ observations of Wiseman \& Ho (1996) implying that the clumps they observed in Orion are surrounded by sheaths that are warmer than the more central parts of the clumps in principle contain information about the energy dissipation in boundary layers between the clumps and a wind.

\section{Global Properties of Mass-Loaded Flows in Clumpy Star Form- ing Regions}

Most studies of the effects of mass, momentum, and energy injection by stars on their surroundings are based on the assumption that the circumstellar material is smoothly distributed. Almost by definition, star forming regions are clumpy. In clumpy media, there is mass, momentum and energy interchange in boundary layers set up at global flow-clump interfaces. The global flow properties, including the scales over which they operate, are affected by this interchange; conversely, the interchange and the clumps themselves are affected by the global flows. Clump material can be fed into a global flow by hydrodynamic ablation, photoevaporation and conduction.

Hydrodynamic ablation is considerably reduced in very subsonic as opposed to supersonic flows (Section 4). Williams, Hartquist \& Dyson (1995) have analysed the dynamics of spherically symmetric, isothermal massloaded winds. Both sub and supersonic flow regions are possible, depending on the distribution of mass-loading centres. However, self-consistent solu- 
tions with Mach number dependent mass injection rates have not yet been obtained. Clearly the response of clumps to stellar winds can be critically affected by their location within the global flow. Unless the wind undergoes a supersonic-subsonic transition and the subsonic region is thick, the range of influence of the wind is not appreciably affected by mass loading since momentum is conserved in the flow

Williams, Dyson \& Redman (1996 and references therein) have considered models for ultracompact HII regions (UCHIIR) in which mass is fed into the HII region by either hydrodynamic ablation or by photoionization. In these models, the HII region is bounded by a stationary recombination front rather than by the usual expanding ionization front. The typical radius of such a region is that characteristic of an UCHIIR i.e. $\sim 0.1 \mathrm{pc}$, which is about two orders of magnitude less than the radius of the initial Strömgren sphere which would be set up in the interclump medium of the RMC. Mass-loading greatly reduces the range of influence of photoionization. If the effects of a stellar wind are included, the scale sizes are unaltered but the Mach number variation in the flow - and therefore the ablation rate - is. If the mass-loaded wind exits supersonically from the UCHIIR (Dyson, Williams \& Redman 1995), any subsequent supersonic pressurisation is effected by a neutral wind moving at most at a few times the ionized gas sound speed rather than at the much higher wind terminal velocity. Mixing at boundary layers between clumps and the global flow involves neutral gas and not highly ionized gas.

The internal structures of supernova remnants are strongly affected by interior mass addition (e.g. McKee \& Ostriker 1977; Chièze and Lazareff 1981; Cowie, McKee \& Ostriker 1981; Dyson \& Hartquist 1987; White \& Long 1991; Arthur \& Henney 1996). Mass addition can enhance radiative cooling. For example, a remnant in the standard uniform density Sedov phase has an integrated energy loss $\propto t^{1.5}$, whereas a remnant hydrodynamically mass-loaded from a uniform distribution of mass-loading sources has an integrated energy loss $\propto t^{4.4}$ (Dyson \& Hartquist 1987). In deriving these dependences, Kahn's (1976) cooling law approximation has been used, which almost certainly has led to an underestimate of the effects of mass-loading on radiative losses as, in reality, the ionization structure in picked-up material requires a significant amount of time to reach equilibrium resulting in an increase in the cooling rate coefficient.

\section{Conclusions}

The presence of stars can induce additional stellar birth only if the range of the ambient medium's response is greater than the distances between the progenitor clumps and the gravitational collapse of these clumps occurs on 
timescales shorter than any evaporation timescales. Unless it is embedded in a very subsonic flow, the ratio of the gravitational collapse and evaporative timescales for a magnetically subcritical dense core, similar to those in which low-mass stars form, is likely to be much greater than the corresponding ratio for a magnetically supercritical clump, like those in which high-mass stars are born. Evaporation of cores or clumps into global flows can lead to supersonic to subsonic transitions in the global flows thus creating regions in which the ablation rate is suppressed resulting in a decreased ratio of the gravitational collapse and evaporation timescale and hence an increased chance of star formation. However, in many cases, mass-loading greatly diminishes the range of response of the ambient medium to a star. The consequences for phenomena such as sequential star formation and superwind generation in starburst galaxies are yet to be explored.

\section{References}

Arons J., Max C. E., 1975, Astrophys.J., 196, L77

Arthur S. J., Henney W. J., 1996, Astrophys.J., 457, 752

Bertoldi F., McKee C. F., 1990, Astrophys.J., 354, 529

Charnley S. B., Dyson J. E., Hartquist T. W., Williams D. A., 1990, Mon.Not.R.ast.Soc., 243, 405

Chièze J. P., Lazareff B., 1981, Astron.EAAstrophys., 95, 194

Cioffi D. F., McKee C. F., Bertschinger E., 1988, Astrophys.J., 334, 252

Cowie L. L., McKee C. F., Ostriker J. P., 1981, Astrophys.J., 247, 908

Dyson J. E., Hartquist T. W., 1987, Mon.Not.R.ast.Soc., 228, 453

Dyson J. E., 1968, Astrophys.Space Sci., 1, 388

Dyson J. E., 1994, in Ray T. P., Beckwith S. V. W., eds, Star Formation and Techniques in Infrared and $\mathrm{mm}$ Astronomy. Springer-Verlag: Berlin, 93

Dyson J. E., Hartquist T. W., Biro S., 1993, Mon.Not.R.ast.Soc., 261, 430

Dyson J. E., Williams R. J. R., Redman M. P., 1995, Mon.Not.R.ast.Soc., 277, 700

Elmegreen B. G., Lada C. J., 1977, Astrophys.J., 214, 725

Elmegreen B. G., 1979, Astrophys.J., 232, 729

Goldsmith P. F., Langer W. D., Wilson R., 1986, Astrophys.J., 303, L11

Hartquist T. W., Dyson J. E., Williams R. J. R., 1997, Astrophys.J., In press

Hartquist T. W., Dyson J. E., Pettini M., Smith L. J., 1986, Mon.Not.R.ast.Soc., 211, 715

Hartquist T. W., Rawlings J. M. C., Williams D. A., Dalgarno A., 1993, Quart.J.R.ast.Soc., 34, 213

Hirahara Y., Suzuki H., Yamamoto S., Kawaguchi K., Ohishi M., Takano S., Ishikawa S. I., Masuda A., 1992, Astrophys.J., 394, 539

Kahn F. D., 1969, Physica, 41, 172

Klein R. I., McKee C. F., Colella P., 1994, Astrophys.J., 420, 213

Kulsrud R., Pearce W. A., 1969, Astrophys.J., 156, 445

Lefloch B., Lazareff B., 1995, Astron.EAstrophys., 289, 559

Lefloch B., Lazareff B., 1996, Astron.EAstrophys., 301, 552

MacLow M. M., McKee C. F., Klein R. I., Stone J. M., Norman M. L., 1994, Astrophys.J., 433, 757

McKee C. F., Ostriker J. P., 1977, Astrophys.J., 215, 213

McKee C. F., 1989, Astrophys.J., 345, 782

Mouschovias T. C., Psaltis D., 1995, Astrophys.J., 444, L105 
Mouschovias T. C., Spitzer Jr. L., 1976, Astrophys.J., 210, 326

Mouschovias T. C., 1987, in Morfill G. E., Scholer M., eds, Physical Processes in Interstellar Clouds. Reidel: Dordrect, 413

Myers P. C., Benson D. J., 1983, Astrophys.J., 266, 309

Myers P. C., 1990, in Hartquist T. W., ed, Molecular Astrophysics - A Volume Honouring Alexander Dalgarno. Cambridge University Press: Cambridge, 328

Nejad L. A. M., Hartquist T. W., 1994, Astrophys.Space Sci., 220, 253

Nittmann J., Falle S. A. E. G., Gaskell P. H., 1982, Mon.Not.R.ast.Soc., 201, 833

Oppenheimer M., Dalgarno A., 1974, Astrophys.J., 192, 29

Rawlings J. M., Hartquist T. W., 1997, Astrophys.J., In press

Redman M. P., Williams R. J. R., Dyson J. E., Hartquist T. W., Fernandez B. R., 1997, Astron. EAstrophys., Submitted

Schilke P., Keene J., Le Bouret J., Pineau des Forêts G., Roueff E. G., 1995, Astron. $\mathcal{G}$ Astrophys., 294, L17

Shu F. H., Adams F. C., Lizano S., 1987, Ann.Rev.Astron.Astrophys., 25, 84

Sutherland R. S., Hartquist T. W., Bally J., Dyson J. E., 1997, Astrophys.J., Submitted

Tedds J. A., Brand P. W. J. L., Brand M. G., Chrysostomou A., Fernandes A. J. L., 1996, in Millar T. J., Raga A. C., eds, Shocks in Astrophysics. Kluwer: Dordrect, 39

Umebayashi T., Nakano T., 1980, PASJ, 32, 405

van Dishoek E. F., 1987, in Vardya M. S., Tarafdar S. P., eds, IAU Symposium 120 Astrochemistry. Reidel: Dordrect, 51

White R. L., Long K. S., 1991, Astrophys.J., 373, 543

Williams J. P., Blitz L., Stark A. A., 1995, Astrophys.J., 451, 252

Williams R. J. R., Dyson J. E., Redman M. P., 1996, Mon.Not.R.ast.Soc., 280, 667

Williams D. A., Hartquist T. W., Caselli P., 1996, Mon.Not.R.ast.Soc., 282, 900

Williams R. J. R., Hartquist T. W., Dyson J. E., 1995, Astrophys.J., 446, 759

Williams D. A., Hartquist T. W., Whittet D. C. B., 1992, Mon.Not.R.ast.Soc., 258, 599

Wiseman J. J., Ho P. T., 1996, Nature, 382, 139

Woodward P. R., 1976, Astrophys.J., 196, 555 